\title{
Introduction of covariance components in slip inversion of land and seafloor geodetic data and application to slip deficit rate estimation in the Nankai Trough subduction zone
}

\author{
Ryoichiro Agata ${ }^{1}$ \\ ${ }^{1}$ Japan Agency for Marine-Earth Science and Technology
}

August 2, 2019

This is a non-peer-reviewed EarthArXiv preprint.

\begin{abstract}
When spatial distribution of observation stations has bias in geodetic slip inversion, modeling errors in the inversion scheme may result in significant unnatural short-wave components in estimated slip distribution, which overfit to data. Combined use of both land and seafloor geodetic data in slip inversion often leads to such situations. To avoid overfitting, I proposed a method to incorporate proper covariance components in the data covariance matrix originated by modeling errors in the geodetic slip inversion. Because linearity of the inversion problem is retained, widely-known approaches to introduce prior constraints to the inversion, which assumes linear inversion, are easily applied to the proposed method. Synthetic tests showed that the introduction of covariance components allowed for the estimation of slip distributions closer to the true one, avoiding overfitting to geodetic data in biasedly distributed observation stations, compared to a conventional approach that does not introduce covariance components. I applied the proposed method to the estimation of the slip deficit rate in the Nankai Trough subduction zone, using geodetic data of displacement rates provided by land GNSS stations and seafloor GNSS-Acoustic stations. The proposed method estimated a reasonably smooth distribution of slip deficit rate compared to the conventional approach. Spatial distribution of residuals in the displacement rates suggested that the proposed method successfully avoids overfitting to the block motion at the south of the Median Tectonic Line, which the physical model I assumed here cannot describe.
\end{abstract}

\section{Introduction}

The development of the observation technique introduced seafloor geodesy such as GNSS (Global Navigation Satellite System)-Acoustic (GNSS-A) techniques and ocean bottom pressure (OBP) gauges to geodetic slip inversion, which was previously performed mainly based on GNSS data. The introduction of seafloor geodesy has drastically increased the resolution of inversions and improved the constraints on coseismic slips (e.g. [13]), afterslips, post-earthquake deformation (e.g. [24, 15, 1]), slow slip events (e.g. [29, 30]), slip deficit rates (e.g. [14, 37]), and plate motion itself (e.g. [4]). However, the combined use of land and seafloor geodetic data often results in the spatially biased distribution of the stations, because seafloor observation is usually more sparse and is located in more limited regions than land observations due to the relatively high cost of installation and operation. If such bias in spatial distribution is not properly considered in the inversion scheme, overfitting to the data sometimes causes unnatural short-wave components in the estimated slip distribution. To avoid this, larger weights are usually put on seafloor data (e.g. $[10,13,7]$ ), or a downsampling of the land stations is carried out 
(e.g. [37]). However, it is generally difficult to objectively determine the parameter for weighting and downsampling.

In the studies of seismic waveform inversions, [32, 33] pointed out that the introduction of proper covariance components in the data covariance matrix, which is often neglected in geodetic slip inversions, can avoid overfitting to biasedly-distributed data. They discussed the sampling rate of the waveform data; they estimated the covariance of data in the time series based on the propagation of modeling errors due to anelastic attenuation of seismic waves and succeeded in the coseismic slip inversion that does not depend on the sampling rate of the waveform data. The same idea should be applicable to the slip inversion of geodetic data with spatial bias by associating covariance components to modeling errors in the inversion scheme.

As shown later, modeling errors in geodetic inversion mainly consist of errors in Green's functions and slip distribution. $[6,21]$ introduced covariance components that depend on model parameters to make estimations, focusing on the error in Green's functions due to factors such as the uncertainty of elastic parameters and dip angles. They formulated a nonlinear inversion based on a Bayesian sampling algorithm, while the geodetic slip inversion is commonly formulated as a linear problem. Here we propose an alternative approach that estimates spatial covariance between each geodetic data based on the Green's function itself. Because this method does not require model parameters to calculate the data covariance matrix, widely accepted linear inversion approaches such as [31] can be applied in the same way used by [32] for seismic waveform inversions.

This paper is organized as follows: Section 2 describes the mathematical formulation. Section 3 presents synthetic tests and compares the proposed method with a conventional approach. In Section 4, I apply the proposed method to the inversion of geodetic data of interseismic displacement rates and show that overfitting to the data is avoided in the estimation of the slip deficit rate when the proposed approach is used. Concluding remarks are provided in Section 5.

\section{Formulation}

\subsection{Calculation of data covariance matrix}

Let us discuss a simple linear inversion problem $\mathbf{u}=\mathbf{G} \mathbf{p}$, where $\mathbf{u}, \mathbf{G}$ and $\mathbf{p}$ are a vector for observation data, an observation (Green's function) matrix to relate the observation and unknowns, and a vector for unknown parameters, respectively. I assume Gaussian distribution of all stochastic variables. In many studies of geodetic inversions, the misfit vector $\varepsilon=\mathbf{u}-\mathbf{G p}$ is considered to obey the Gaussian distribution, i.e., $\boldsymbol{\varepsilon} \sim N(\mathbf{0}, \mathbf{E})$, where $\mathbf{E}$ is a diagonal matrix. In such cases, covariance components in the data covariance matrix are totally ignored. However, based on more detailed analysis, $\varepsilon$ can be decomposed as

$$
\begin{aligned}
\varepsilon & =\mathbf{u}-\mathbf{G} \mathbf{p} \\
& =\mathbf{u}-\boldsymbol{G}_{\text {true }}\left(\mathbf{p}_{\text {true }}\right)+\boldsymbol{G}_{\text {true }}\left(\mathbf{p}_{\text {true }}\right)-\mathbf{G} \mathbf{p} \\
& =\varepsilon_{\mathrm{o}}+\boldsymbol{\varepsilon}_{\mathrm{m}},
\end{aligned}
$$

where $\varepsilon_{\mathrm{o}}=\mathbf{u}-\boldsymbol{G}_{\text {true }}\left(\mathbf{p}_{\text {true }}\right)$ and $\boldsymbol{\varepsilon}_{\mathrm{m}}=\boldsymbol{G}_{\text {true }}\left(\mathbf{p}_{\text {true }}\right)-\mathbf{G p}$ are error terms due to observation and modeling, respectively. How to set $\varepsilon_{\mathrm{o}}$ is relatively well studied $[9,14$,$] , and the use of a diagonal$ matrix for the covariance matrix is also expected to be a fairly good approximation. Hereafter, let us suppose the proper probabilistic distribution function (PDF) for $\varepsilon_{\mathrm{o}} \sim N\left(\mathbf{0}, \sigma_{\mathrm{o}}^{2} \mathbf{E}_{\mathrm{o}}\right)$ is known. $[33,6,21]$ assumed that errors of Green's function is the main source of the error term $\varepsilon_{\mathrm{m}}$. If so, it is obvious that $\varepsilon_{\mathrm{m}}$ has stronger spatial correlation between each component. Simply put, [33,6,21] assumed $\boldsymbol{\varepsilon}_{\mathrm{m}}=\boldsymbol{G}_{\text {true }}\left(\mathbf{p}_{\text {true }}\right)-\mathbf{G} \mathbf{p}=\delta \mathbf{G} \mathbf{p}$, where $\delta \mathbf{G}$ is error of Green's function. Such a manner requires estimating error of Green's function and performing nonlinear inversion because the data covariance matrix changes with the model parameters p. Instead, in this study, I assume

$$
\begin{aligned}
\varepsilon_{\mathrm{m}} & =\boldsymbol{G}_{\text {true }}\left(\mathbf{p}_{\text {true }}\right)-\mathbf{G} \mathbf{p} \\
& \simeq \mathbf{G}\left(\mathbf{p}_{\text {true }}^{\prime}-\mathbf{p}\right),
\end{aligned}
$$


where $\mathbf{p}_{\text {true }}^{\prime}=\mathbf{G}^{+} \mathbf{G}_{\text {true }} \mathbf{p}_{\text {true }}$, and $\mathbf{G}^{+}$and $\mathbf{G}_{\text {true }}$ is a general inverse matrix of $\mathbf{G}$ and a matrix that linearizes the nonlinear operator $\boldsymbol{G}_{\text {true }}$, respectively. Here, I use an approximation that $\mathbf{G G}^{+} \simeq \mathbf{I}$, where $\mathbf{I}$ is an identity matrix. Let us assume a simple PDF for $\delta \mathbf{p}=\mathbf{p}_{\text {true }}^{\prime}-\mathbf{p}$, such as $\delta \mathbf{p} \sim N\left(\mathbf{0}, \sigma_{\mathrm{s}}^{2} \mathbf{E}_{\mathrm{s}}\right)$, where I use $\mathbf{E}_{\mathrm{s}}=\mathbf{I}$, for instance. Then, due to the propagation law of uncertainty,

$$
\boldsymbol{\varepsilon}_{\mathrm{m}} \simeq \mathbf{G} \delta \mathbf{p} \sim N\left(\mathbf{0}, \sigma_{\mathrm{s}}^{2} \mathbf{G E}_{\mathrm{s}} \mathbf{G}^{\mathrm{T}}\right) .
$$

Because $\mathbf{G}$ is usually a non-sparse matrix, $\sigma_{\mathrm{s}}^{2} \mathbf{G} \mathbf{E}_{\mathrm{s}} \mathbf{G}^{\mathrm{T}}$ has non-zero covariance components. Based on the additivity of the normal distribution, the likelihood function of $\mathbf{u}$ can be written as

$$
P\left(\mathbf{u} \mid \mathbf{p}, \sigma_{\mathrm{s}}, \sigma_{\mathrm{o}}\right)=(2 \pi)^{-N / 2}\|\mathbf{E}\|^{-1 / 2} \exp \left[-\frac{1}{2}(\mathbf{u}-\mathbf{G} \mathbf{p})^{\mathrm{T}} \mathbf{E}^{-1}(\mathbf{u}-\mathbf{G p})\right]
$$

where $\mathbf{E}=\sigma_{\mathrm{s}}^{2} \mathbf{G E}_{\mathrm{s}} \mathbf{G}^{\mathrm{T}}+\sigma_{\mathrm{o}}^{2} \mathbf{E}_{\mathrm{o}}$. Note that covariance components are introduced to the likelihood function only by calculating matrix-matrix products using $\mathbf{G}$ without any additional information on the inverse problem. Furthermore, because the linear property of the inverse problem is not changed, the proposed approach is easily applied to existing geodetic inversion methods such as [31].

As mentioned above, Equation 5 assumes $\mathbf{G G}^{+}=\mathbf{I}$, which holds when $\mathbf{G}^{\prime}$ s number of columns is greater than the number of rows. Otherwise the equality is just an approximation. The effect of this approximation on the estimation results will be discussed in Section 4 . In addition, $\mathbf{E}_{\mathrm{s}}=\mathbf{I}$ implicitly assumes $\mathbf{G}^{+} \mathbf{G}_{\text {true }} \simeq \mathbf{I}$. I should note that an excessive difference between $\mathbf{G}$ and $\mathbf{G}_{\text {true }}$ may reduce the estimation accuracy of the prior PDF.

\subsection{Applicability to the inversion formulation based on Bayes' theorem and Akaike's Bayesian information criterion}

I demonstrate the applicability of the proposed approach to an existing geodetic linear analysis method proposed by [31]. Their method incorporated prior constraints of the smoothness of slip distribution to the observation equations, constructing a Bayesian model with unknown hyperparameters. The values of the hyperparameters, which control the structure of the Bayesian model, are objectively determined from observation data by minimizing Akaike's Bayesian information criterion (ABIC) [2, ]. I can directly apply the likelihood function defined in Equation 7 to their approach. I first assume a prior PDF with respect to the smoothness of the slip distribution,

$$
P\left(\mathbf{p} \mid \sigma_{\mathrm{p}}\right)=(2 \pi)^{-P / 2}\left\|\frac{1}{\sigma_{\mathrm{p}}^{2}} \boldsymbol{\Lambda}\right\| \|^{1 / 2} \exp \left[-\frac{1}{2} \mathbf{p}^{\mathrm{T}}\left(\frac{1}{\sigma_{\mathrm{p}}^{2}} \mathbf{L}^{\mathrm{T}} \mathbf{L}\right) \mathbf{p}\right],
$$

where $\mathbf{L}$ is a matrix corresponding to a discretized Laplacian operator, $P$ is the rank of $\mathbf{L}^{\mathrm{T}} \mathbf{L}$, and $\boldsymbol{\Lambda}$ is a $P \times P$ diagonal matrix that has non-zero eigenvalues of $\mathbf{L}^{\mathrm{T}} \mathbf{L}$ as its diagonal components. Based on Bayes' theorem, the posterior PDF of the model parameters is written as

$$
\begin{aligned}
P\left(\mathbf{p} \mid \mathbf{u}, \sigma_{\mathrm{s}}, \sigma_{\mathrm{o}}, \sigma_{\mathrm{p}}\right) & \sim P\left(\mathbf{u} \mid \mathbf{p}, \sigma_{\mathrm{s}}, \sigma_{\mathrm{o}}\right) P\left(\mathbf{p} \mid \sigma_{\mathrm{p}}\right) \\
& \left.\sim(2 \pi)^{-(N+P) / 2}\|\mathbf{E}\|^{-1 / 2}\left\|\frac{1}{\sigma_{\mathrm{p}}^{2}} \boldsymbol{\Lambda}\right\|\right|^{1 / 2} \exp \left[-\frac{1}{2} s(\mathbf{p})\right],
\end{aligned}
$$

where

$$
s(\mathbf{p})=(\mathbf{u}-\mathbf{G} \mathbf{p})^{\mathrm{T}} \mathbf{E}^{-1}(\mathbf{u}-\mathbf{G} \mathbf{p})+\mathbf{p}^{\mathrm{T}}\left(\frac{1}{\sigma_{\mathrm{p}}^{2}} \mathbf{L}^{\mathrm{T}} \mathbf{L}\right) \mathbf{p}
$$

Optimal model parameters that minimize this functional is readily obtained once the hyperparameters are determined, as

$$
\mathbf{p}^{*}=\left(\mathbf{G}^{\mathrm{T}} \mathbf{E}^{-1} \mathbf{G}+\frac{1}{\sigma_{\mathrm{p}}^{2}} \mathbf{L}^{\mathrm{T}} \mathbf{L}\right)^{-1} \mathbf{G}^{\mathrm{T}} \mathbf{E}^{-1} \mathbf{u}
$$


Here, I introduce ABIC, which is defined as

$$
\mathrm{ABIC}=(-2) \ln L\left(\sigma_{\mathrm{s}}^{2}, \sigma_{\mathrm{o}}^{2}, \sigma_{\mathrm{p}}^{2}\right)
$$

where

$$
L\left(\sigma_{\mathrm{s}}^{2}, \sigma_{\mathrm{o}}^{2}, \sigma_{\mathrm{p}}^{2}\right)=\int P\left(\mathbf{u} \mid \mathbf{p}, \sigma_{\mathrm{s}}, \sigma_{\mathrm{o}}\right) P\left(\mathbf{p} \mid \sigma_{\mathrm{p}}\right) d \mathbf{p} .
$$

The necessary condition for ABIC to be minimized is

$$
\frac{\partial L\left(\sigma_{\mathrm{s}}^{2}, \sigma_{\mathrm{o}}^{2}, \sigma_{\mathrm{p}}^{2}\right)}{\partial \sigma_{\mathrm{s}}^{2}}=0,
$$

which allows for ABIC being dependent on two hyperparameters (See Appendix A for detailed calculation of ABIC). I adopt the hyperparameters that minimize ABIC in the inversion, performing minimization using a grid search. Thus, I can determine the hyperparameters for the PDF for the proposed method by applying [31].

\section{Synthetic test}

In this section, I perform synthetic tests of geodetic inversion that include significant modeling errors and compare results obtained using the proposed method and a conventional one. When using the conventional method, I assume $\varepsilon \sim N\left(\mathbf{0}, \sigma^{2} \mathbf{E}\right)$, where $\sigma$ is a hyperparameter and $\mathbf{E}$ is a diagonal matrix. The approach of [31] is used elsewhere, determining the hyperparameter on the prior PDF of the parameter based on ABIC in the same way as described in Section 2.2. I show the advantage of introducing covariance components due to modeling errors in the geodetic inversion analyses.

\subsection{Problem setting}

I consider a dip slip fault in the elastic homogeneous half-space, which models a plate boundary. The fault geometry is shown in Figure 1 (a): the fault width $W=680 \mathrm{~km}$, the fault length $L=340 \mathrm{~km}$, and the dip angle $\theta=40^{\circ}$, respectively. I consider a synthetic test problem of estimating slip deficit distribution (SDR), which is targeted in the application of actual data in Section 4. SDR and geodetic data for inversion have the dimension of velocity. Otherwise, it is the same as in ordinary slip inversions. The true SDR distribution shown in Figure 1 (b) is inputted in Plane 1 and elastic responses in virtual observation points are used as the synthetic data. The virtual observation points are biasedly distributed, which mimics geodetic inversion using both land and seafloor stations. Here, I consider two patterns of observation distribution, the main difference of which is the location of the seafloor stations, as shown by red and green points in Figure 1 (b). I assume that 16 relatively isolated points located direcly above the main source area are seafloor stations and 800 other points land stations. Following the configuration of the real world problem presented in Section 4, I use the $x, y$ and $z$ components of the displacement rates in the land stations, and the $x$ and $y$ for the seafloor stations. I add artificial Gaussian noise to the computed displacements, for which the standard deviations are $2.5 \times 10^{-4} \mathrm{~m} / \mathrm{yr}$ for the horizontal component and $5.0 \times 10^{-4} \mathrm{~m} / \mathrm{yr}$ for the vertical component. The covariance matrix for the observation error, $\mathbf{E}_{\mathrm{o}}$ for the proposed method and $\mathbf{E}$ for the conventional method, is a diagonal matrix, for which the components are set according to these noise levels. To introduce modeling errors in the synthetic tests, I calculate Green's functions in Plane 2, a fault plane that has a perturbation dip angle $d \theta$ from the true one (Figure 1 (a)). The size of subfaults $h$ to input unit slip for calculating Green's functions is $20 \mathrm{~km}$. I consider $d \theta=2^{\circ}, 5^{\circ}$ and $10^{\circ}$ for comparison. I calculate both the synthetic data and Green's functions using the computation program of [20]. Using the synthetic data and the Green's functions, I perform inversion analyses using the proposed method and the conventional method and compare the estimated SDR distribution. 


\subsection{Results}

When $d \theta=2^{\circ}$, the estimation results look almost identical, regardless of which method is used (Figure 2 ). It implies that when modeling errors are small, neglecting covariance components does not cause significant differences in the estimation results. When $d \theta=5^{\circ}$, modeling errors become larger than in the case of $d \theta=2^{\circ}$. As a result, we observe significant differences in the estimation results (Figure 3 ). The proposed method estimates a smoother SDR distribution, which is closer to the true one than the conventional method; the root mean square error (RMSE) of the estimated slip deficit distribution is $3.4 \times 10^{-3} \mathrm{~m} / \mathrm{yr}$ for the proposed method and $7.0 \times 10^{-3} \mathrm{~m} / \mathrm{yr}$ for the conventional one. Such a tendency becomes more obvious when $d \theta=10^{\circ}$; many significant short-wave components, for which the spatial distribution seems strongly affected by the locations of observation stations, are seen in the estimation result of the conventional method, which are not included in that of the proposed one (Figure 4). Although the larger error in Green's functions also degrades the estimation results of the proposed method with SDR peaks imposed to the region along the trench axis, the basic feature of the true SDR, which consists of two peaks, remains in the Figure 4 (a). The RSME of the SDR distribution in the case of the proposed method is $1.2 \times 10^{-2} \mathrm{~m} / \mathrm{yr}$, which is significantly smaller than that for the conventional method, $1.7 \times 10^{-2} \mathrm{~m} / \mathrm{yr}$. Such tendency strongly appears when a more biased distribution of observation stations is adopted for $d \theta=10^{\circ}$ (Figure 5). The amplitude of short-wave components in the SDR estimated by the conventional method is even larger in this case, whereas the proposed method still estimates a smooth distribution regardless of the location of the stations. A relatively large discrepancy between observed and simulated displacements shown in Figure 5 (c)(e) implies that overfitting to the data is avoided in the case of the proposed method.

In summary, the proposed method gives smoother solutions that have smaller RMSE to the true SDR, compared to the conventional method. When the modeling errors are small, the proposed method provides almost identical estimation results to that of the conventional method. These findings suggests that the proposed method succeeds in estimating the modeling error and PDFs, which produces favorable estimation results.

\section{Application to slip deficit rate estimation in Nankai Trough sub- duction zone}

The Nankai Trough subduction zone, located off the coast of southwest Japan, is well known for great earthquakes with recurrence intervals of 100-200 years [3, ]. To estimate the accumulated stress on the focal region in the present day, it is important to estimate the SDR on the plate boundary. The interseismic displacement rates in southwest Japan have been compiled by continuous GNSS observation provided by GNSS Earth Observation Network System (GEONET), which is managed by the Geospatial Information Authority of Japan [22, ]. In addition, seafloor geodetic observation using GNSS-A has provided interseismic displacement rates in the region directly above the megathrust $[37$,$] . While combined use of the land and seafloor geodetic data is promising in increasing spatial$ resolution of SDR estimation compared to the case solely using the land data, spatial bias in the distribution of observation stations may lead to a severe problem of overfitting as discussed in Section 1. To address this problem, I apply the proposed method to the estimation of SDR in the Nankai Trough subduction zone and discuss its validity.

\subsection{Data and modeling setting}

I use GNSS and GNSS-A data of the displacement rates, which were estimated by [18] with a common reference frame fixed to the Eurasian plate. The GNSS displacement rates were estimated for the east, north and up components for the period 2005-2009. The GPS-A displacement rates were estimated for the east and north components for various period between 2004 to 2012 depending on the station. From the original data set, I extracted the data in the station located in the area between $131.5^{\circ} \mathrm{E}-$ $138.5^{\circ} \mathrm{N}$ and $31.5^{\circ} \mathrm{N}-35^{\circ} \mathrm{N}$, which are located reasonably near to the target fault region (Figure 6). 
As a result, 316 GNSS stations and 19 GNSS-A stations are used. I use homogeneous elastic halfspace to calculate Green's functions. It means that the displacement rate data is expected to include at least two major sources of modeling errors; one is displacement due to rigid block motions. The Median Tectonic Line (MTL) is a famous arc-parallel fault system in this region, in which the rightlateral strike-slip movement is related to the oblique subduction of the Philippine Sea plate. Past studies estimated that this movement imposes around $5 \mathrm{~mm} / \mathrm{yr}$ motion in the southern block of MTL $[17,25$,$] . Another factor is the 3D heterogeneous elastic structure that cannot be incorporated in$ the homogeneous elastic half-space. Plate boundary geometry is extracted from "Japan integrated velocity structure model version 1" (JVSM) [16, ], a unified velocity structure model for the Japanese Islands. Plate boundary is discretized by $4 \mathrm{~km}$-sized triangles. I use 3rd-order bicubic B-splines for basis function to discretize the slip deficit distribution following [31], central points of which are in $20 \mathrm{~km}$ intervals (Figure 6). I locate 432 central points, so that they discretize the area of the plate boundary between $131.5^{\circ} \mathrm{E}-139^{\circ} \mathrm{N}, 31.5^{\circ} \mathrm{N}-35^{\circ} \mathrm{N}$, and shallower than $60 \mathrm{~km}$ depth (Figure 6 ). Because we estimate SDR in two components (the direction of plate convergence and the one perpendicular to it), the number of unknowns is 864 . Response function due to a triangular fault in the elastic homogeneous half-space is calculated based on an analytical expression of elastic response due to an angular dislocation proposed by [5].

In the inversion scheme, I assume $\mathbf{E}_{\mathrm{o}}=\mathbf{I}$ for the proposed method and $\mathbf{E}=\mathbf{I}$ for the conventional method for simplicity.

\subsection{Results and Discussions}

Figure 7 (a) shows the SDR distribution estimated using the proposed method. A smooth solution, which is not strongly correlated with the positions of geodetic stations, is obtained. In contrast, SDR distribution estimated by the conventional method has significant short-wave components directly below the area in which geodetic stations are absent (Figure 7 (b)). The maximum slip deficit rate in this subduction zone is expected to be no more than the reported plate convergence rate, i.e., at most around $6 \mathrm{~cm} / \mathrm{yr}[11$,$] . This knowledge in plate tectonics is more consistent to the estimation result of$ the proposed method. It follows that the proposed method avoids overfitting to the factors that are not modeled in the scheme, which otherwise become sources of unnatural short-wave components in the estimated slip. ABIC is $-9.658 \times 10^{3}$ for the proposed method and $-9.541 \times 10^{3}$ for the conventional method, which suggests that the SDR model estimated using the proposed method is more preferred in terms of model selection as well. Here are the detailed discussions:

\subsubsection{Residuals and the Median Tectonic Line}

Figure 8 compares the residuals of surface horizontal displacement rate between the proposed and conventional methods. Systematic patterns are observed in the result of the proposed method. Part of these residuals are expected to originate from the displacement rates due to factors that are not modeled in the scheme. In particular, we observe a significant change of residuals around MTL and the Beppu-Shibabara graben (BSG), west of MTL; significant displacement rates towards the west are seen in the land station at the south of MTL and BSG, but are not seen at the north. These residuals in the west direction in the southern block of MTL are at around the rate of $5 \mathrm{~mm} / \mathrm{yr}$, which is consistent to the implication on block motion in the southern block of MTL from past studies [17, 25, ]. This strongly indicates that our method succeeds in avoiding overfitting to the displacement rates produced by relative motion along MTL and BSG. Such systematic residuals around MTL and BSG are not seen in the results of the conventional one. However, we also see strong systematic patterns in other regions such as the Tokai area, for which I cannot suggest a reasonable explanation. While the proposed method is expected to reduce the effect of the modeling errors, it is, needless to say, also important to make efforts to reduce modeling errors such as the consideration of block motion $[18,19$, ] and incorporation of 3D elastic heterogeneity and viscoelasticity [27, 19, ]. 


\subsubsection{Resolution of estimated SDR}

Figure 9 shows the resolution of the SDR estimations. The resolution I discuss here is the diagonal component of the model resolution matrix. In our formulation, the model resolution matrix $\mathbf{R}_{\mathrm{m}}$ is computed as:

$$
\mathbf{R}_{\mathrm{m}}=\left(\mathbf{G}^{\mathrm{T}} \mathbf{E}^{-1} \mathbf{G}+\frac{1}{\sigma_{\mathrm{p}}^{2}} \mathbf{L}^{\mathrm{T}} \mathbf{L}\right)^{-1} \mathbf{G}^{\mathrm{T}} \mathbf{E}^{-1} \mathbf{G}
$$

The L-2 norm of the resolution for two direction of slips in each subfault is plotted. Interestingly, the resolution of the SDR distribution estimated by the proposed method is significantly low. This is reasonable because the larger weight put on the smoothness constraint, the lower resolution obtained in the estimation result, shown in Equation 16. This indicates that we cannot succeed in both avoiding overfitting and achieving high-resolution estimation when the problem includes significant modeling errors.

\subsubsection{Data downsampling and data covariance components}

One of the important issues in the combined use of land and seafloor data for conventional geodetic slip inversion is how to determine the weight on each kind of data; some previous studies place larger weight on seafloor data $[13$,$] or downsample the land stations [37$,$] to seek a better balance between$ the land and seafloor data. One advantage of the proposed method is that introducing proper data covariance prevents us from determining weights on the data. Figure 10 shows estimation results with 100 randomly sampled land stations out of the original set of 316 stations. The slip distributions estimated using the proposed method are not sensitive to the number of land stations used in the estimation, whereas it is greatly affected when using the conventional method. Table 1 lists RSME in each case of estimation. In addition, downsampling of the land stations does not affect the RMSE much regarding both all and the seafloor data for the proposed method, while it affects significantly for the conventional one. It follows that the introduction of the proper data covariance matrix avoids the problem of determining weights on each kind of observation data in the cost function of an inversion problem.

When all the stations are used in the inversion, G's number of rows is $316 \times 3+19 \times 2=986$, which is greater than the number of columns, 832. This means $\mathbf{G G}^{+}=\mathbf{I}$, which Equation 5 in Session 2 assumes, just approximately holds. In the case of downsampling, G's number of rows is $100 \times 3+19 \times 2=338$, which is smaller than the number of columns, with which $\mathbf{G G}^{+}=\mathbf{I}$ holds. In spite of such a difference, the estimated SDR distributions in the two cases is similar, which suggests that the approximation $\mathbf{G G}^{+} \simeq \mathbf{I}$ in the former case does not affect much the estimation result.

\section{Conclusion}

I proposed a method to incorporate proper covariance components originated by modeling errors in geodetic slip inversion to avoid overfitting to geodetic data in biasedly distributed observation stations. Because this method retains linearlity of the inverse problem, it is applicable to widelyknown approaches to introduce prior constraint to the inversion, such as the method of [31]. I carried out synthetic tests of SDR estimation in a dip slip fault, in which I introduce modeling error in estimation using different dip angles to the true fault geometry. The proposed method provided smoother solutions that have smaller RMSE to the true SDR assumed in the synthetic test, compared to the conventional method. I applied the proposed method to estimation of SDR in the Nankai Trough subduction zone, using geodetic data of displacement rates provided by land GNSS stations and seafloor GNSS-Acoustic stations. The proposed method estimated a reasonably smooth estimation result compared to a conventional approach that does not introduce covariance components. Spatial distribution of residual in displacement rates suggested that the proposed method successfully avoids overfitting to block motion at the south of Median Tectonic Line, which the model that I assumed 
(a)

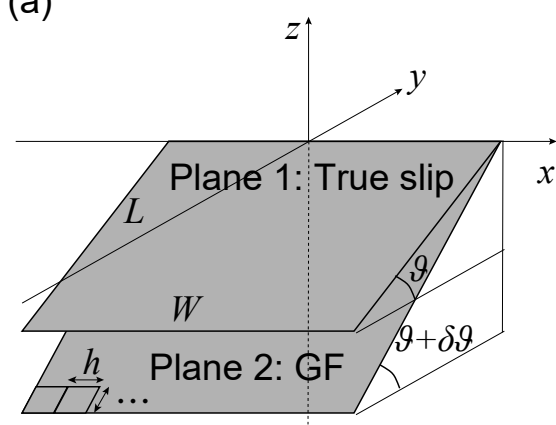

(b)

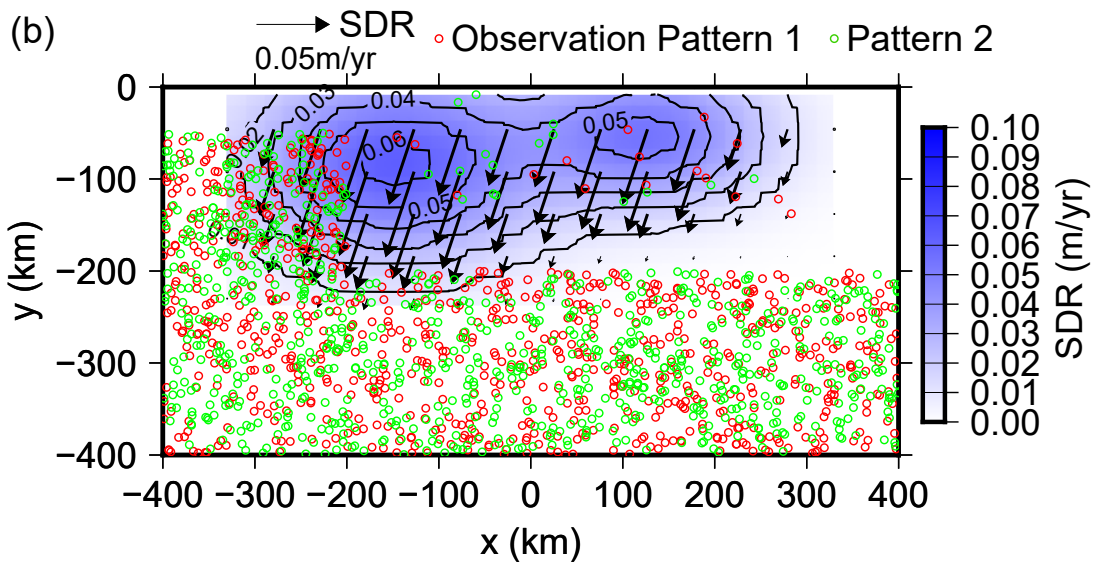

Figure 1: Problem setting of the synthetic tests. (a) Fault geometry. The fault width $W=680 \mathrm{~km}$, the fault length $L=340 \mathrm{~km}$ and the dip angle $\theta=40^{\circ}$, respectively. The true SDR distribution shown in (b) is input in Plane 1. Green's functions are calculated in Plane 2, a fault plane that has a perturbation dip angle $d \theta$ from Plane 1 . The size of subfaults $h$ for calculating Green's functions is $20 \mathrm{~km}$. I consider $d \theta=2^{\circ}, 5^{\circ}$ and $10^{\circ}$ for comparison. (b) The true SDR distribution input in Plane 1. Red and green points show two different patterns of observation locations. I assume that 16 relatively isolated points are seafloor stations and 800 other points land stations.

Table 1: Comparison of RMSE of the displacement rates in the estimation results in the four cases. "Original" and "downsample" denote the cases with 316 and 100 land stations, respctively.

\begin{tabular}{c|cc} 
& Total & Seafloor \\
Case & RMSE $(\mathrm{m} / \mathrm{yr})$ & RMSE $(\mathrm{m} / \mathrm{yr})$ \\
\hline Conventional/Original & 0.00129 & 0.00234 \\
Conventional/Downsampled & 0.00169 & 0.00300 \\
Proposed/Original & 0.00336 & 0.00469 \\
Proposed/Downsampled & 0.00334 & 0.00444 \\
\hline
\end{tabular}

here cannot describe. It was also shown that it is difficult to succeed in both avoiding overfitting and achieving high-resolution estimation when the problem includes significant modeling error. Estimation results using downsampled land data shows that the estimation results obtained using the proposed method are robust to a change in sparseness of the stations, which suggests that the proposed method does not require subjective determination of weight in each data.

As indicated by the examples in seismic waveform inversions [32, 33, ], the proposed approach should be also effective in time-dependent geodetic slip inversion, such as post-earthquake slip inversion considering viscoelasticity [34, ]. Introduction of proper covariance components in time is also expected to allow for robust estimation results to irregular time intervals in time series of seafloor geodetic data from GNSS-Acoustic stations (e.g. [28, 26]). However, increase of the number of unknowns in time-dependent inversion may lead to difficulty in calculating inverse of the covariance matrix, which requires further consideration in computation algorithm.

Although the proposed method can reduce the effect of modeling errors in estimation results, the best way to improve the inversion results is of course to reduce the modeling errors itself by revising the numerical model. Recent development of numerical simulation and high-performance computing methods is a key factor for this purpose $[12,8$,$] . In the future work, more direct consideration$ of uncertainty in Green's function is desirable, which should be introduced by executing multiple computations of crustal deformation $[35,36$,$] .$ 


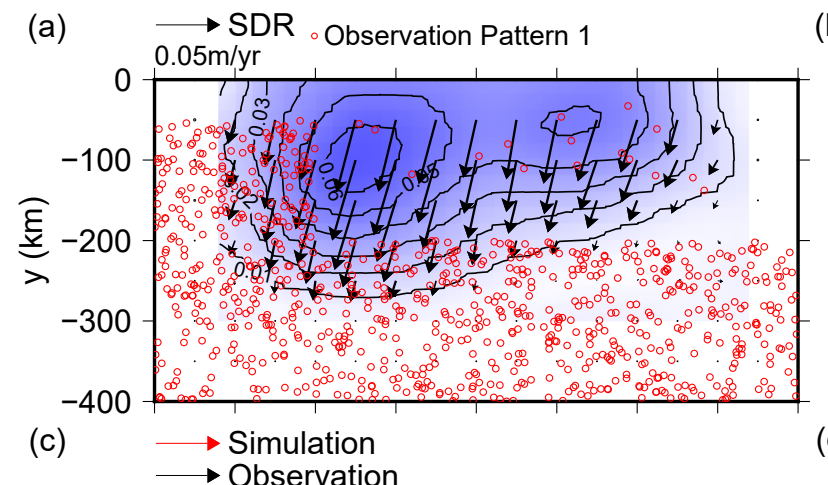

(b) $\underset{0.05 \mathrm{~m} / \mathrm{yr}}{\mathrm{SDR}}$ 。Observation Pattern 1
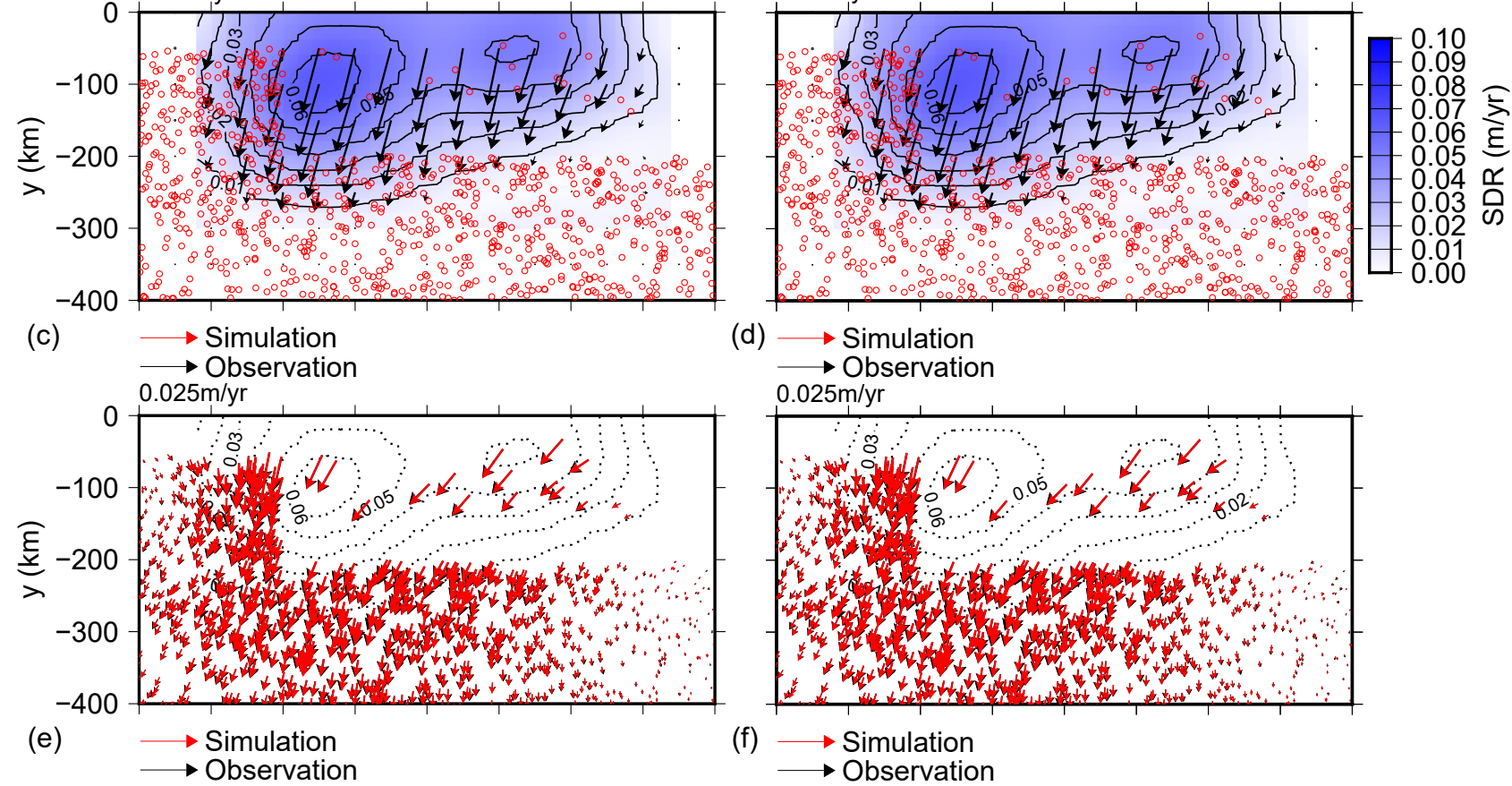

d) $\longrightarrow$ Simulation
$\longrightarrow$ Observation $0.025 \mathrm{~m} / \mathrm{yr}$

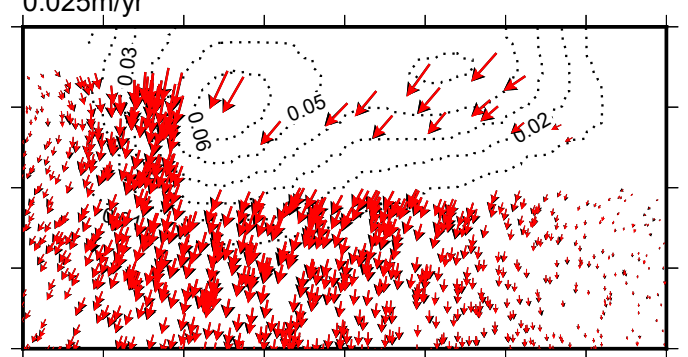

(f) $\longrightarrow$ Simulation

$\longrightarrow$ Observation
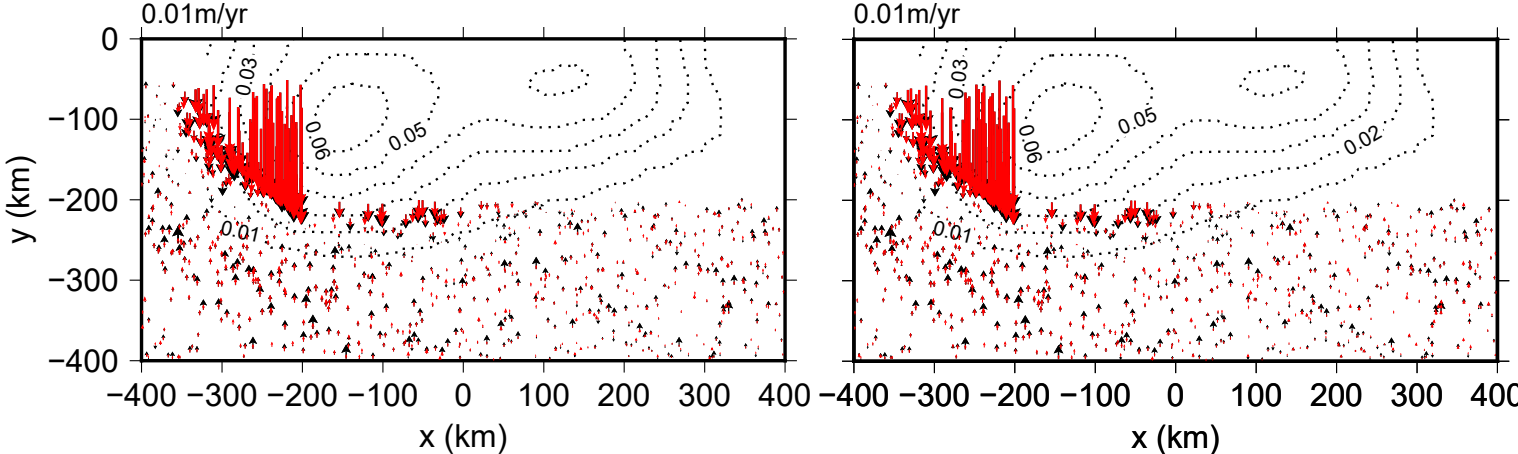

Figure 2: Results of the synthetic test with $d \theta=2^{\circ}$. (a)(b) SDR distribution estimated using the proposed and conventional methods. (c)(d) Comparison of horizontal components in artificially observed displacement rates and simulated one based on the estimated SDR distribution. (e)(f) Comparison of vertical components. 


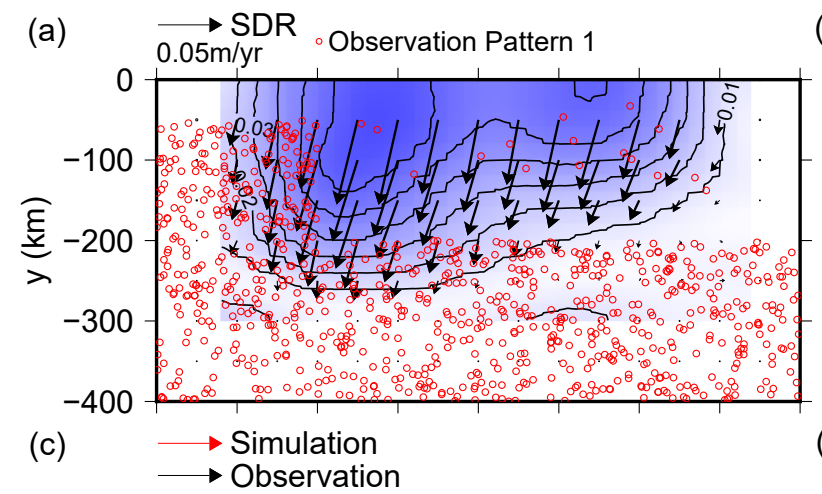

(b) $\underset{0.05 \mathrm{~m} / \mathrm{yr}}{\mathrm{S}} \quad$ Observation Pattern 1
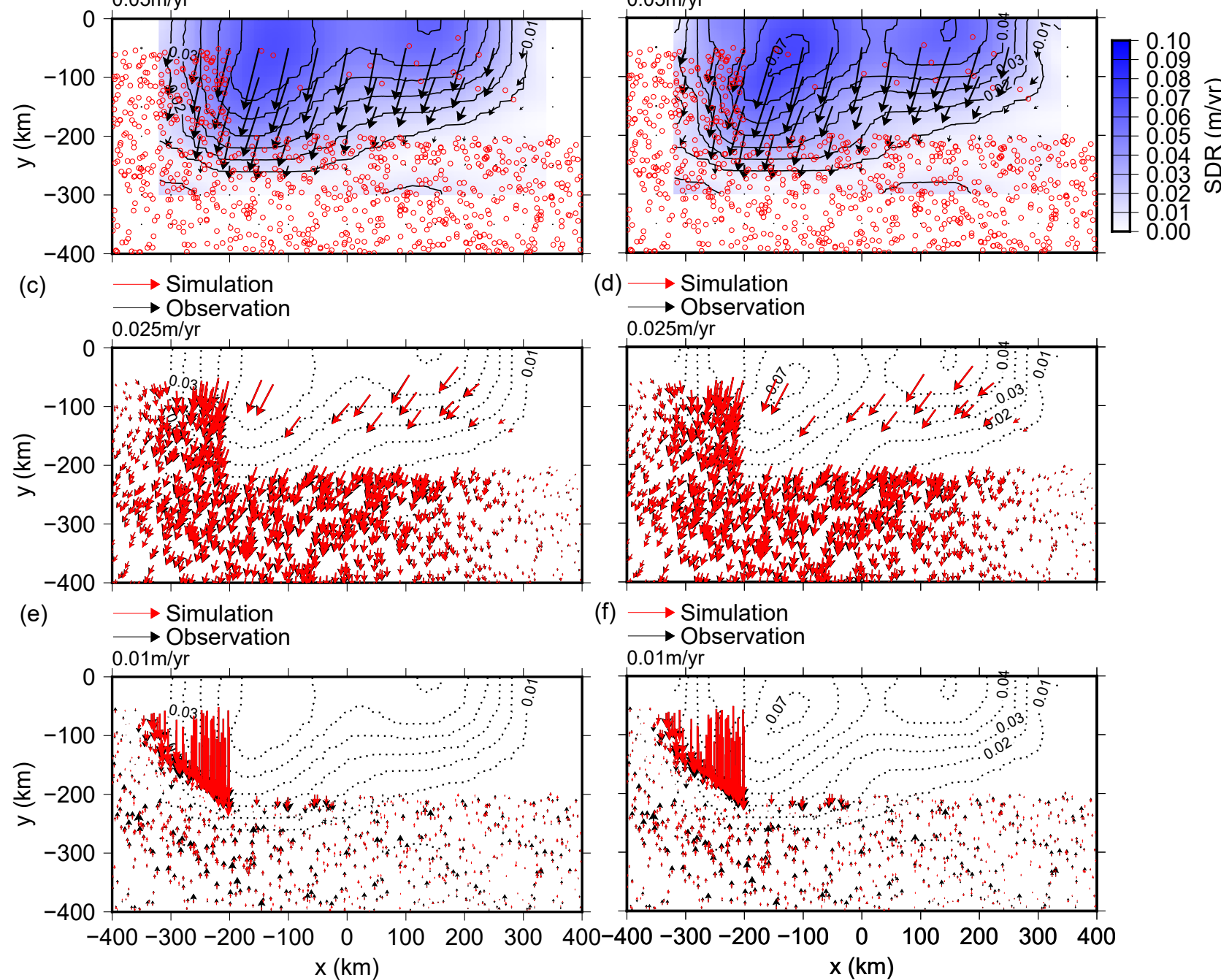

(d) $\longrightarrow$ Simulation

$\longrightarrow$ Observation

$0.025 \mathrm{~m} / \mathrm{yr}$

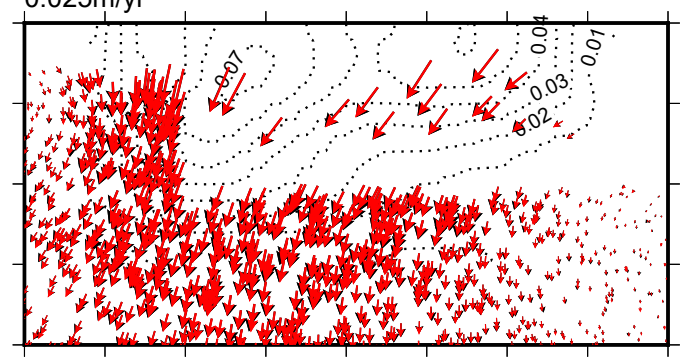

(f) $\longrightarrow$ Simulation

$\longrightarrow$ Observation

$0.01 \mathrm{~m} / \mathrm{yr}$



Figure 3: Results of the synthetic test with $d \theta=5^{\circ}$. (a)(b) SDR distribution estimated using the proposed and conventional methods. (c)(d) Comparison of horizontal components in artificially observed displacement rates and simulated one based on the estimated SDR distribution. (e)(f) Comparison of vertical components. 


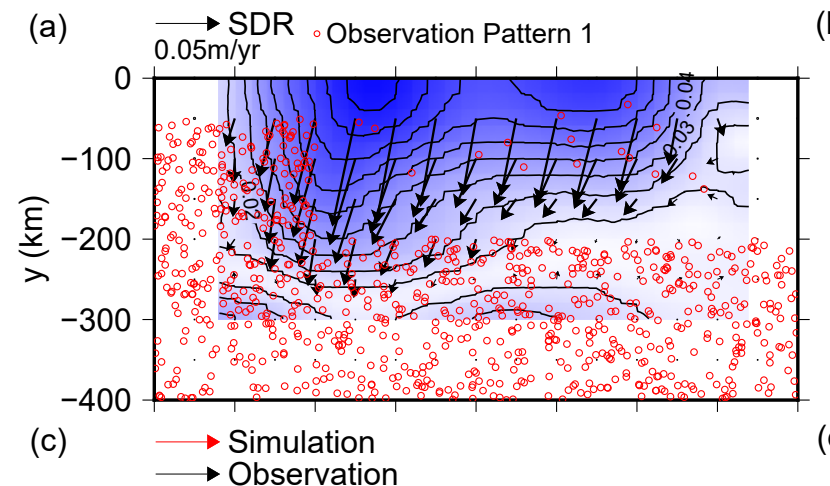

(b) $\underset{0.05 \mathrm{~m} / \mathrm{yr}}{\mathrm{SDR}}$ 。Observation Pattern 1

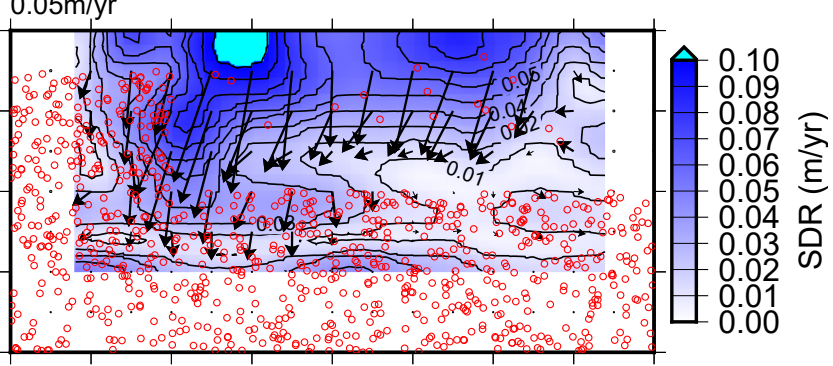

(d) $\longrightarrow$ Simulation

$\longrightarrow$ Observation

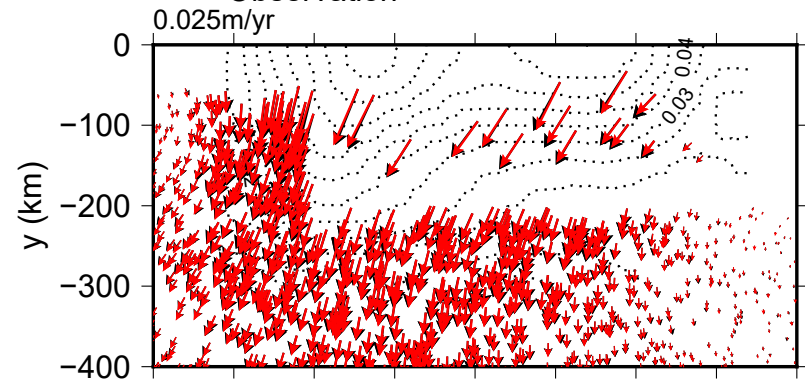

(e) $\quad \begin{aligned} & \longrightarrow \text { Simulation } \\ & \longrightarrow \text { Observation } \\ & 0.01 \mathrm{~m} / \mathrm{yr}\end{aligned}$

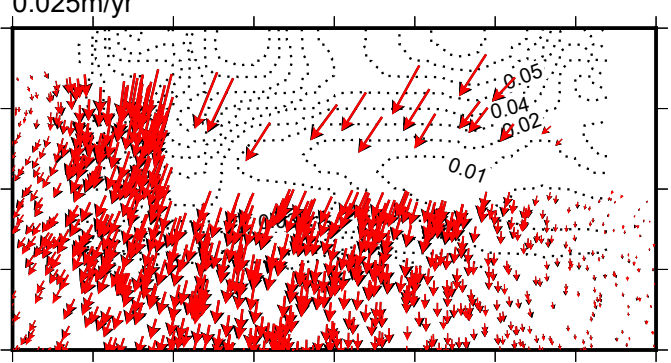

(f) $\longrightarrow$ Simulation

$\longrightarrow$ Observation
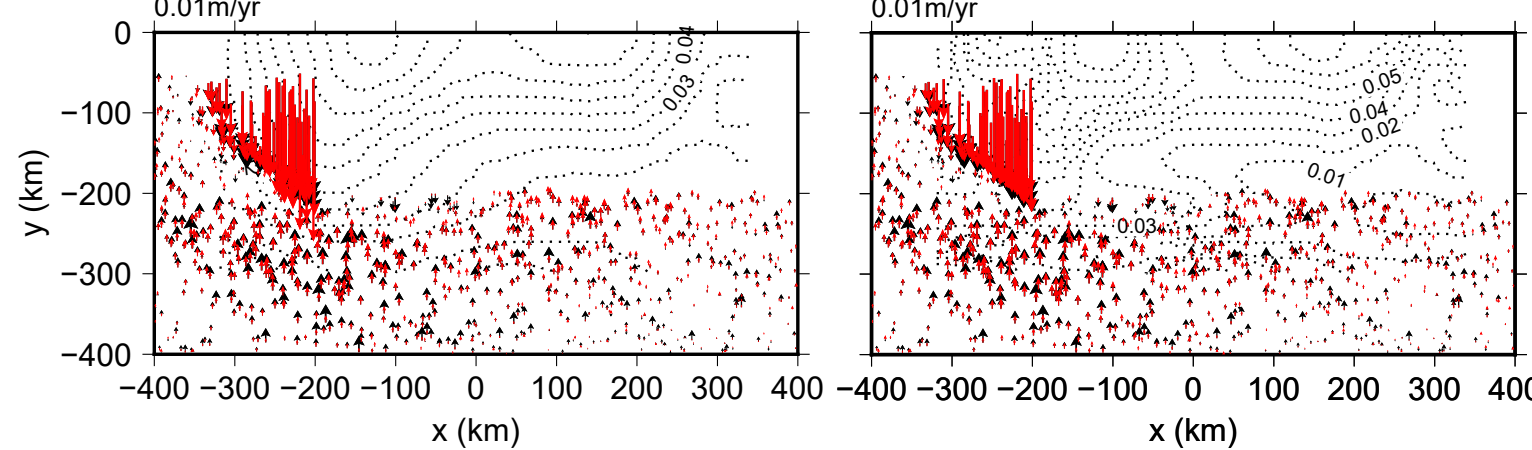

Figure 4: Results of the synthetic test with $d \theta=10^{\circ}$. (a)(b) SDR distribution estimated using the proposed and conventional methods. (c)(d) Comparison of horizontal components in artificially observed displacement rates and simulated one based on the estimated SDR distribution. (e)(f) Comparison of vertical components. 




(b) $\underset{0.05 \mathrm{~m} / \mathrm{yr}}{\mathrm{SDR}}$. Observation Pattern 2
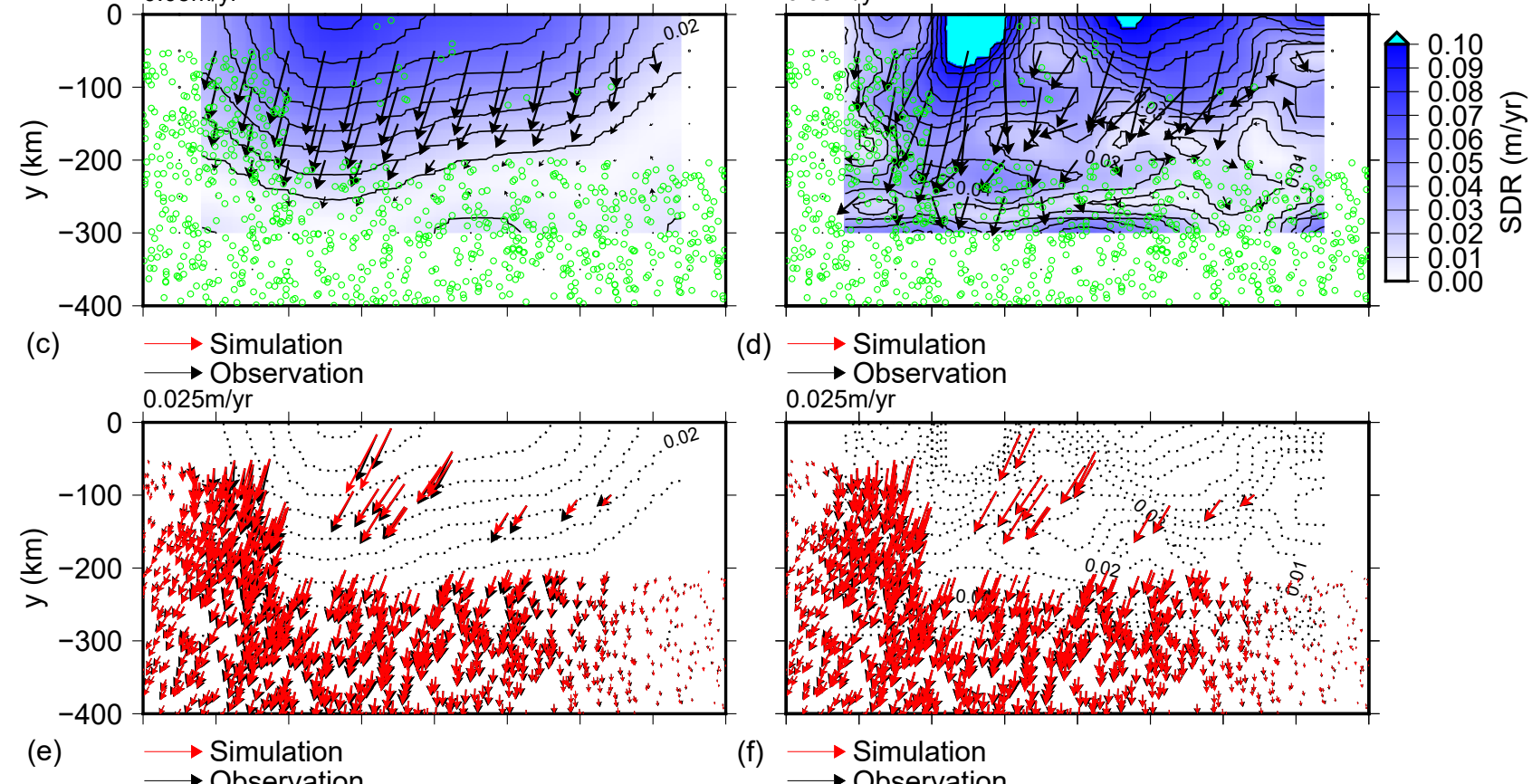

(d) $\longrightarrow$ Simulation

$\longrightarrow$ Observation

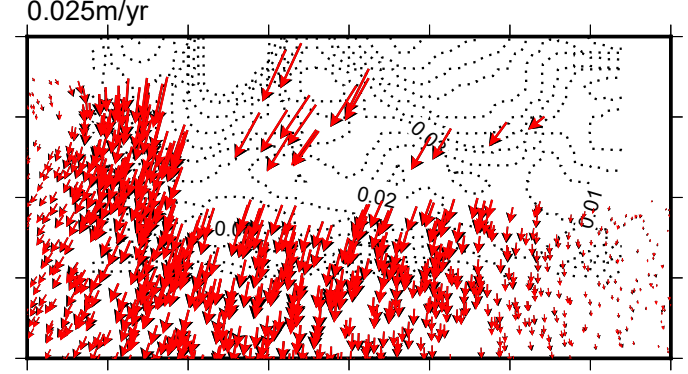

(e) $\quad \longrightarrow$ Simulation

(f) $\longrightarrow$ Simulation

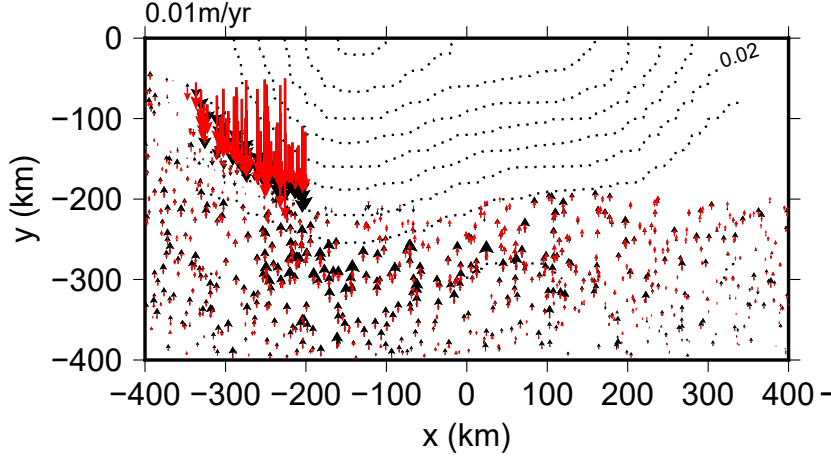

$\rightarrow$ Observation $0.01 \mathrm{~m} / \mathrm{yr}$

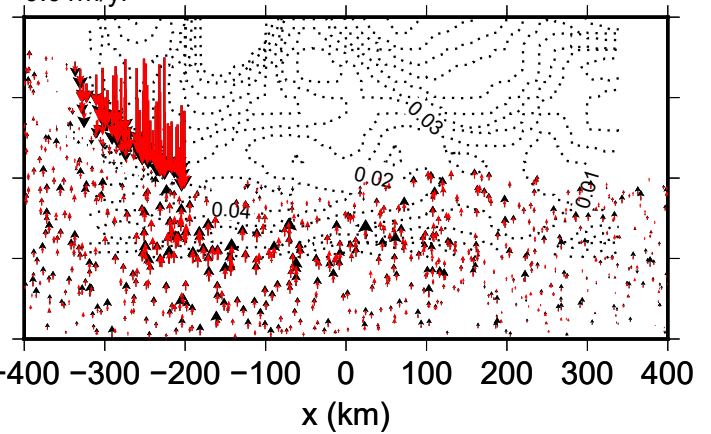

Figure 5: Results of synthetic test with $d \theta=10^{\circ}$ with observation Pattern 2. (a)(b) SDR distribution estimated using the proposed and conventional methods. (c)(d) Comparison of horizontal components in artificially observed displacement rates and simulated ones based on the estimated SDR distribution. (e)(f) Comparison of vertical components. 


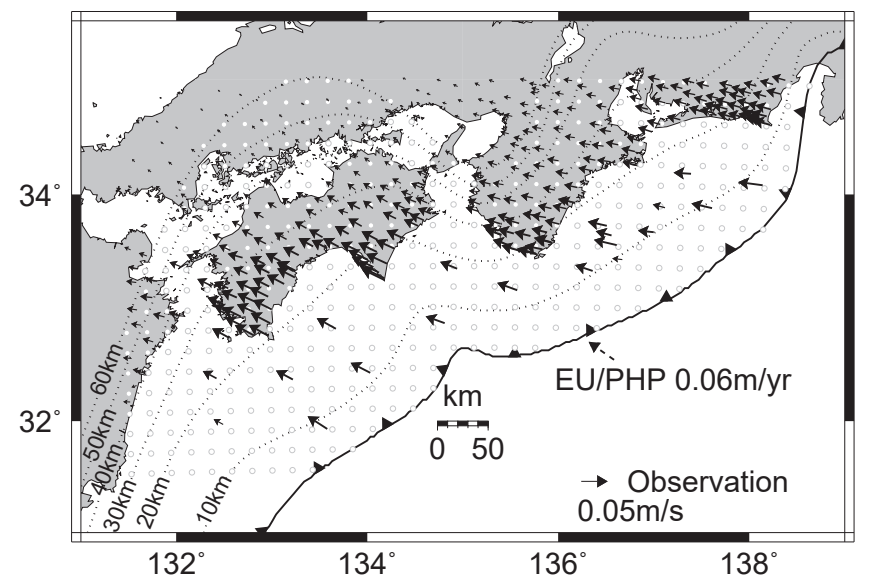

Figure 6: Tectonic setting in the Nankai Trough subduction zone. The dotted contour lines denote the iso-depth of the plate boundary. The arrows denote the displacement rate estimated by [18]. The white dots are the central points of bicubic B-spline unit faults to calculate Green's functions.
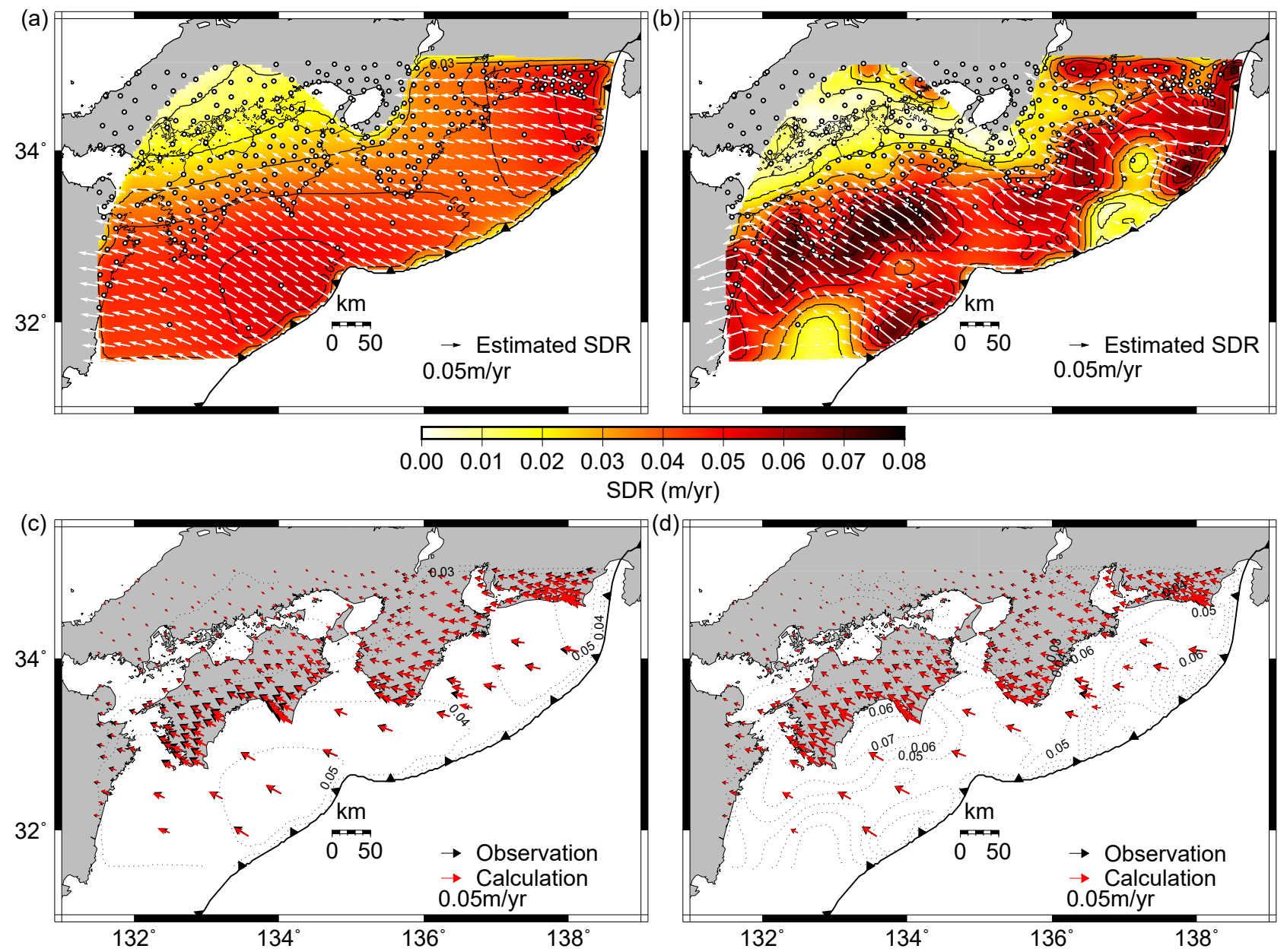

Figure 7: Estimated interseismic SDR distribution. (a)(b) SDR distribution estimated using the proposed and conventional method, respectively. The white arrows and color contours are the vectors and the norm of SDR, respectively. The white dots denote the location of the observation. (c)(d) A comparison of horizontal components in the observed displacement rates and the simulated one based on the estimated SDR distribution. The dotted lines denote the norm of SDR with $1 \mathrm{~cm} / \mathrm{yr}$ interval. 

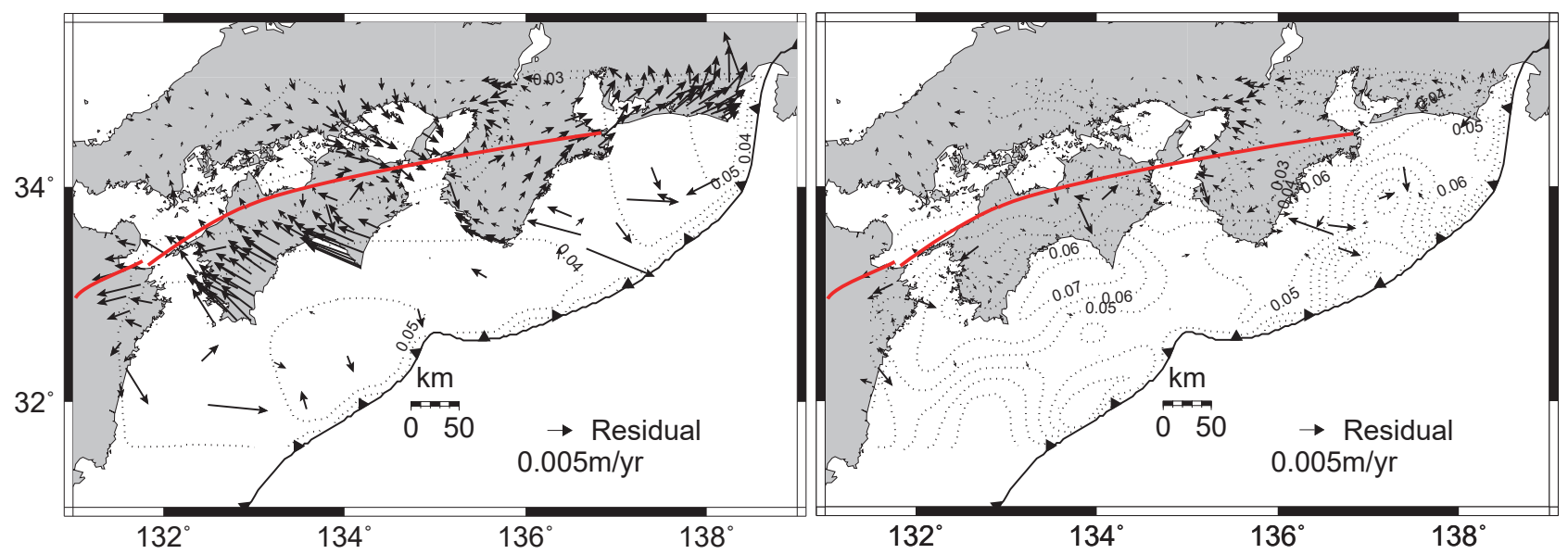

Figure 8: Residuals (difference between observed and simulated displacement rates) in (a) the proposed and (b) the conventional method. The arrows are the residual vectors. The dotted lines denote the norm of SDR with $1 \mathrm{~cm} / \mathrm{yr}$ interval. The red lines denote the location of MTL and BSG.
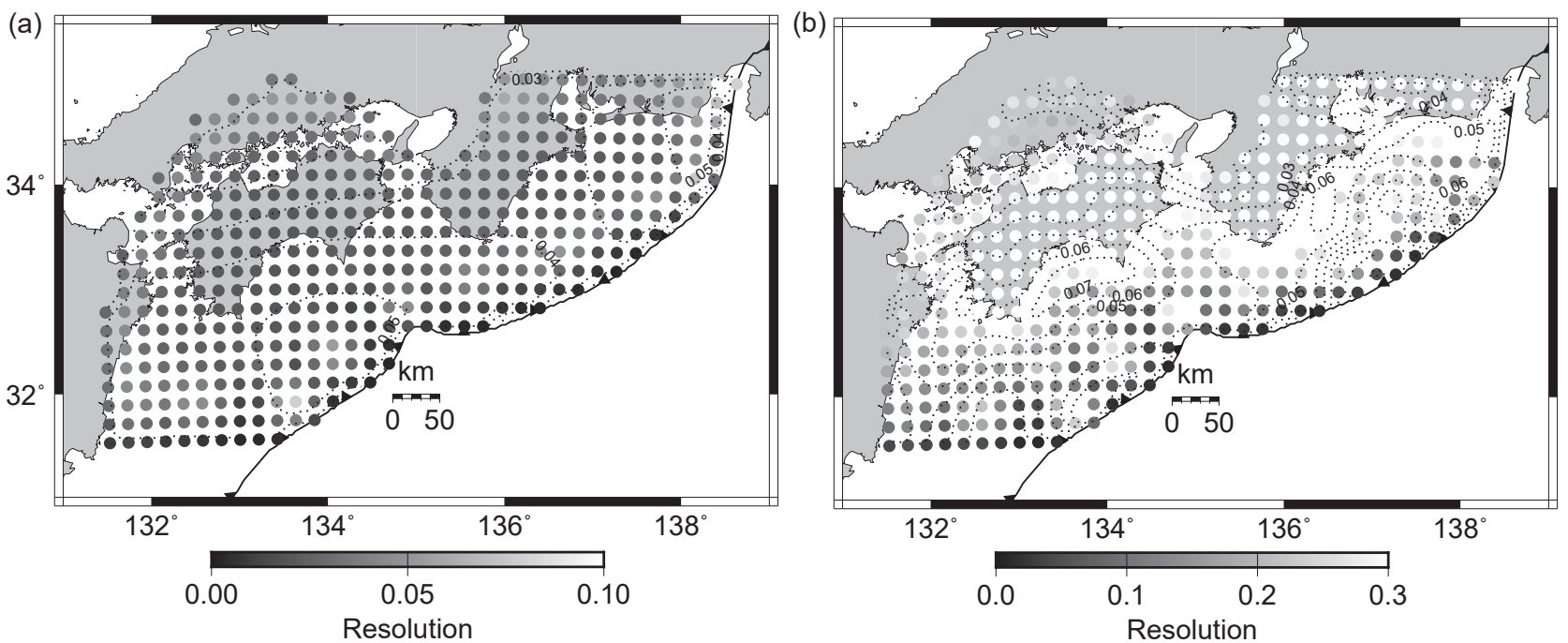

Figure 9: The resolution in the estimation results for the case with (a) the proposed and (b) the conventional method. The resolution I discuss here is the diagonal component of the model resolution matrix. Plotted is the L-2 norm of the resolution for the two directions of slips in each subfault. Note that the color scales are different between (a) and (b). 

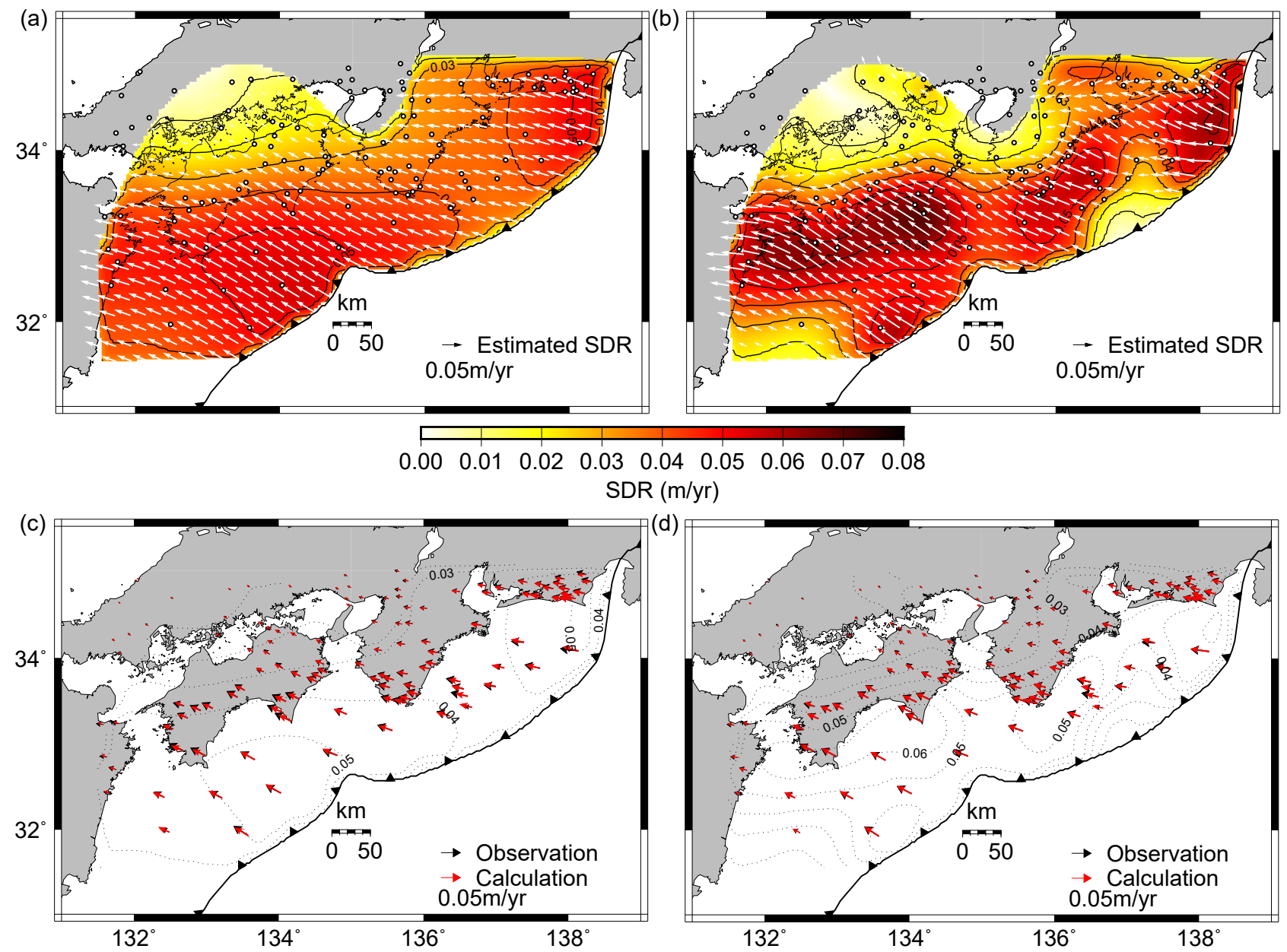

Figure 10: Estimated interseismic SDR distribution with downsampled land observation. (a)(b) SDR distribution estimated using the proposed and conventional method, respectively. The white arrows and color contours are the vectors and the norm of SDR, respectively. The white dots denote the location of observation. (c)(d) Comparison of horizontal component in the observed displacement rates and the simulated one based on the estimated SDR distribution. Dotted lines denote the norm of SDR with $1 \mathrm{~cm} / \mathrm{yr}$ interval. 


\section{Acknowledgement}

I greatly appreciate the comments on model selection by Dr. Shin-ichi Ito and those on the problem setting of the synthetic tests by Dr. Takeshi Iinuma. This study was supported by JSPS KAKENHI Grant Numbers 19H04631. Some figures were produced using GMT software [23, ].

\section{References}

[1] Ryoichiro Agata, Sylvain D. Barbot, Kohei Fujita, Mamoru Hyodo, Takeshi Iinuma, Ryoko Nakata, Tsuyoshi Ichimura, and Takane Hori. Rapid mantle flow with power-law creep explains deformation after the 2011 tohoku mega-quake. Nature Communications, 10(1):1385, 2019.

[2] T Akaike. Likelihood and Bayes procedure. Bayesian Statistics, pages 143-166, 1980.

[3] Masataka Ando. Source mechanisms and tectonic significance of historical earthquakes along the Nankai Trough, Japan. Tectonophysics, 27(2):119-140, 1975.

[4] C. David Chadwell and Fred N. Spiess. Plate motion at the ridge-transform boundary of the south Cleft segment of the Juan de Fuca Ridge from GPS-Acoustic data. Journal of Geophysical Research, 113(B4):B04415, apr 2008.

[5] Maria Comninou and John Dundurs. The angular dislocation in a half space. Journal of Elasticity, 5(3-4):203-216, 1975.

[6] Zacharie Duputel, Piyush S Agram, Mark Simons, Sarah E Minson, and James L Beck. Accounting for prediction uncertainty when inferring subsurface fault slip. Geophysical Journal International, 197(1):464-482, 2014.

[7] Andrew M Freed, Akinori Hashima, Thorsten W Becker, David A Okaya, Hiroshi Sato, and Yuki Hatanaka. Resolving depth-dependent subduction zone viscosity and afterslip from postseismic displacements following the 2011 tohoku-oki, japan earthquake. Earth and Planetary Science Letters, 459:279-290, 2017.

[8] Kohei Fujita, Tsuyoshi Ichimura, Kentaro Koyama, Hikaru Inoue, Muneo Hori, and Lalith Maddegedara. Fast and scalable low-order implicit unstructured finite-element solver for earth's crust deformation problem. In Proceedings of the Platform for Advanced Scientific Computing Conference, page 11. ACM, 2017.

[9] Yukitoshi Fukahata and Tim J Wright. A non-linear geodetic data inversion using ABIC for slip distribution on a fault with an unknown dip angle. Geophysical Journal International, 173(2):353$364,2008$.

[10] Jun'ichi Fukuda and Kaj M Johnson. Mixed linear-non-linear inversion of crustal deformation data: Bayesian inference of model, weighting and regularization parameters. Geophysical Journal International, 181(3):1441-1458, 2010.

[11] Kosuke Heki and Shin'ichi Miyazaki. Plate convergence and long-term crustal deformation in central japan. Geophysical Research Letters, 28(12):2313-2316, 2001.

[12] Tsuyoshi Ichimura, Ryoichiro Agata, Takane Hori, Kazuro Hirahara, Chihiro Hashimoto, Muneo Hori, and Yukitoshi Fukahata. An elastic/viscoelastic finite element analysis method for crustal deformation using a 3-D island-scale high-fidelity model. Geophysical Journal International, 206(1):114-129, 2016. 
[13] T Iinuma, R Hino, M Kido, D Inazu, Y Osada, Y Ito, M Ohzono, H Tsushima, S Suzuki, H Fujimoto, and S Miura. Coseismic slip distribution of the 2011 off the Pacific Coast of Tohoku Earthquake (M9.0) refined by means of seafloor geodetic data. Journal of Geophysical Research, 117(B7):1-18, jul 2012.

[14] Takeshi Iinuma, Ryota Hino, Motoyuki Kido, Yusaku Ohta, and Satoshi Miura. Interplate Coupling in and Around the Rupture Area of the 2011 Tohoku Earthquake (M9.0) Before Its Occurrence Based on Terrestrial and Seafloor Geodetic Observations. In Manabu Hashimoto, editor, International Symposium on Geodesy for Earthquake and Natural Hazards (GENAH), pages 1119, Cham, 2017. Springer International Publishing.

[15] Takeshi Inuma, Ryota Hino, Naoki Uchida, Wataru Nakamura, Motoyuki Kido, Yukihito Osada, and Satoshi Miura. Seafloor observations indicate spatial separation of coseismic and postseismic slips in the 2011 Tohoku earthquake. Nature communications, 7:13506, 2016.

[16] Kazuki Koketsu, Hiroe Miyake, and Haruhiko Suzuki. Japan integrated velocity structure model version 1. Proceedings of the 15th World Conference on Earthquake Engineering, (1773), 2012.

[17] Shin'ichi Miyazaki and Kosuke Heki. Crustal velocity field of southwest Japan: Subduction and arc-arc collision. Journal of Geophysical Research: Solid Earth, 106(B3):4305-4326, 2001.

[18] Takuya Nishimura, Yusuke Yokota, Keiichi Tadokoro, and Tadafumi Ochi. Strain partitioning and interplate coupling along the northern margin of the philippine sea plate, estimated from global navigation satellite system and global positioning system-acoustic data. Geosphere, 14(2):535$551,2018$.

[19] Akemi Noda, Tatsuhiko Saito, and Eiichi Fukuyama. Slip-deficit rate distribution along the Nankai trough, southwest Japan, with elastic lithosphere and viscoelastic asthenosphere. Journal of Geophysical Research: Solid Earth, 123(9):8125-8142, 2018.

[20] Yoshimitsu Okada. Internal deformation due to shear and tensile faults in a half-space. Bulletin of the Seismological Society of America, 82(2):1018-1040, 1992.

[21] Théa Ragon, Anthony Sladen, and Mark Simons. Accounting for uncertain fault geometry in earthquake source inversions-I: theory and simplified application. Geophysical Journal International, 214(2):1174-1190, 2018.

[22] Takeshi Sagiya. Interplate coupling in the kanto district, central japan, and the boso peninsula silent earthquake in may 1996. Pure and Applied Geophysics, 11(161):2327-2342, 2004.

[23] WHF Smith and P Wessel. Gridding with continuous curvature splines in tension. Geophysics, 55(3):293-305, 1990.

[24] Tianhaozhe Sun, Kelin Wang, Takeshi Iinuma, Ryota Hino, Jiangheng He, Hiromi Fujimoto, Motoyuki Kido, Yukihito Osada, Satoshi Miura, Yusaku Ohta, and Yan Hu. Prevalence of viscoelastic relaxation after the 2011 Tohoku-oki earthquake. Nature, 514:84-87, sep 2014.

[25] Takao Tabei, Manabu Hashimoto, Shin’ichi Miyazaki, Kazuro Hirahara, Fumiaki Kimata, Takeshi Matsushima, Torao Tanaka, Yasuhide Eguchi, Takashi Takaya, Yoshinobu Hoso, et al. Subsurface structure and faulting of the Median Tectonic Line, southwest Japan inferred from GPS velocity field. Earth, planets and space, 54(11):1065-1070, 2002.

[26] Fumiaki Tomita, Motoyuki Kido, Yusaku Ohta, Takeshi Iinuma, and Ryota Hino. Alongtrench variation in seafloor displacements after the 2011 tohoku earthquake. Science Advances, 3(7):e1700113, 2017. 
[27] Shun-ichi Watanabe, Yehuda Bock, Diego Melgar, and Keiichi Tadokoro. Tsunami scenarios based on interseismic models along the Nankai trough, Japan, from seafloor and onshore geodesy. Journal of Geophysical Research: Solid Earth, 123(3):2448-2461, 2018.

[28] Shun'ichi Watanabe, Mariko Sato, Masayuki Fujita, Tadashi Ishikawa, Yusuke Yokota, Naoto Ujihara, and Akira Asada. Evidence of viscoelastic deformation following the 2011 Tohoku-oki earthquake revealed from seafloor geodetic observation. Geophysical Research Letters, aug 2014.

[29] Charles A Williams and Laura M Wallace. Effects of material property variations on slip estimates for subduction interface slow-slip events. Geophysical Research Letters, 42(4):1113-1121, 2015.

[30] Charles A Williams and Laura M Wallace. The impact of realistic elastic properties on inversions of shallow subduction interface slow slip events using seafloor geodetic data. Geophysical Research Letters, 45(15):7462-7470, 2018.

[31] T Yabuki and M Matsu'ura. Geodetic data inversion using a Bayesian information criterion for spatial distribution of fault slip. Geophysical Journal International, 109(2):363-375, 1992.

[32] Yuji Yagi and Yukitoshi Fukahata. Importance of covariance components in inversion analyses of densely sampled observed data: an application to waveform data inversion for seismic source processes. Geophysical Journal International, 175(1):215-221, 2008.

[33] Yuji Yagi and Yukitoshi Fukahata. Rupture process of the 2011 Tohoku-oki earthquake and absolute elastic strain release. Geophysical Research Letters, 38(19):1-5, 2011.

[34] Shuji Yamagiwa, Shin'ichi Miyazaki, Kazuro Hirahara, and Yukitoshi Fukahata. Afterslip and viscoelastic relaxation following the 2011 tohoku-oki earthquake $\left(M_{\mathrm{w}} 9.0\right)$ inferred from inland gps and seafloor gps/acoustic data. Geophysical Research Letters, 42(1):66-73, 2015.

[35] Takuma Yamaguchi, Kohei Fujita, Tsuyoshi Ichimura, Takane Hori, Muneo Hori, and Lalith Wijerathne. Fast Finite Element Analysis Method Using Multiple GPUs for Crustal Deformation and its Application to Stochastic Inversion Analysis with Geometry Uncertainty. Procedia Computer Science, 108:765-775, 2017.

[36] Takuma Yamaguchi, Tsuyoshi Ichimura, Yuji Yagi, Ryoichiro Agata, Takane Hori, and Muneo Hori. Fast crustal deformation computing method for multiple computations accelerated by a graphics processing unit cluster. Geophysical Journal International, 210(2):787-800, 2017.

[37] Yusuke Yokota, Tadashi Ishikawa, Shun-ichi Watanabe, Toshiharu Tashiro, and Akira Asada. Seafloor geodetic constraints on interplate coupling of the Nankai Trough megathrust zone. $\mathrm{Na}$ ture, 534(7607):374, 2016. 


\section{Appendix}

The explicit expression for the likelihood function that consists ABIC is

$$
\begin{aligned}
L\left(\sigma_{\mathrm{s}}^{2}, \sigma_{\mathrm{o}}^{2}, \sigma_{\mathrm{p}}^{2}\right)= & \int P\left(\mathbf{u} \mid \mathbf{p}, \sigma_{\mathrm{s}}, \sigma_{\mathrm{o}}\right) P\left(\mathbf{p} \mid \sigma_{\mathrm{p}}\right) d \mathbf{p} \\
= & (2 \pi)^{-(N+P) / 2}\|\mathbf{E}\|^{-1 / 2}\left\|\frac{1}{\sigma_{\mathrm{p}}^{2}} \mathbf{\Lambda}\right\|^{1 / 2} \exp \left[-\frac{1}{2} s^{\prime}\left(\mathbf{p}^{*}\right) / \sigma_{\mathrm{s}}^{2}\right] \\
& \int \exp \left[-\left(\mathbf{p}-\mathbf{p}^{*}\right)^{\mathrm{T}}\left(\mathbf{G}^{\mathrm{T}} \mathbf{E}^{-1} \mathbf{G}+\frac{1}{\sigma_{\mathrm{p}}^{2}} \mathbf{L}^{\mathrm{T}} \mathbf{L}\right)\left(\mathbf{p}-\mathbf{p}^{*}\right) / 2\right] d \mathbf{p} \\
= & (2 \pi)^{-(N+P) / 2}\|\mathbf{E}\|^{-1 / 2}\left\|\frac{1}{\sigma_{\mathrm{p}}^{2}} \mathbf{\Lambda}\right\|^{1 / 2} \exp \left[-\frac{1}{2} s^{\prime}\left(\mathbf{p}^{*}\right) / \sigma_{\mathrm{s}}^{2}\right] \\
& (2 \pi)^{M / 2} *\left\|\mathbf{G}^{\mathrm{T}} \mathbf{E}^{-1} \mathbf{G}+\frac{1}{\sigma_{\mathrm{p}}^{2}} \mathbf{L}^{\mathrm{T}} \mathbf{L}\right\|^{-1 / 2} \\
= & (2 \pi)^{-(N+P) / 2} \frac{1}{\sigma_{\mathrm{s}}^{N}}\left\|\mathbf{E}^{\prime}\right\|^{-1 / 2} \frac{1}{\sigma_{\mathrm{s}}^{P}}\left\|\frac{1}{\beta^{2}} \mathbf{\Lambda}\right\|^{1 / 2} \exp \left[-\frac{1}{2} s^{\prime}\left(\mathbf{p}^{*}\right) / \sigma_{\mathrm{s}}^{2}\right] \\
& (2 \pi)^{M / 2} * \sigma_{\mathrm{s}}^{M}\left\|\mathbf{G}^{\mathrm{T}} \mathbf{E}^{\prime-1} \mathbf{G}+\frac{1}{\beta^{2}} \mathbf{L}^{\mathrm{T}} \mathbf{L}\right\|^{-1 / 2}
\end{aligned}
$$

where

$$
s^{\prime}(\mathbf{p})=(\mathbf{u}-\mathbf{G} \mathbf{p})^{\mathrm{T}} \mathbf{E}^{\prime-1}(\mathbf{u}-\mathbf{G} \mathbf{p})+\frac{1}{\beta^{2}} \mathbf{L}^{\mathrm{T}} \mathbf{L},
$$

$\mathbf{E}^{\prime}=\mathbf{G E}_{\mathrm{s}} \mathbf{G}^{\mathrm{T}}+\alpha^{2} \mathbf{E}_{\mathrm{o}}, \alpha=\sigma_{\mathrm{o}} / \sigma_{\mathrm{s}}$, and $\beta=\sigma_{\mathrm{p}} / \sigma_{\mathrm{s}}$. To carry out the integration, I utilize that $s(\mathbf{p})$ can be expressed using $\mathbf{p}^{*}$ the optimal value because of linearity, as

$$
s(\mathbf{p})=s\left(\mathbf{p}^{*}\right)+\left(\mathbf{p}-\mathbf{p}^{*}\right)^{\mathrm{T}}\left(\mathbf{G}^{\mathrm{T}} \mathbf{E}^{-1} \mathbf{G}+\frac{1}{\sigma_{\mathrm{p}}^{2}} \mathbf{L}^{\mathrm{T}} \mathbf{L}\right)\left(\mathbf{p}-\mathbf{p}^{*}\right) .
$$

As discussed in the main text, the necessary condition for ABIC to be minimized is

$$
\frac{\partial L\left(\sigma_{\mathrm{s}}^{2}, \sigma_{\mathrm{o}}^{2}, \sigma_{\mathrm{p}}^{2}\right)}{\partial \sigma_{\mathrm{s}}^{2}}=0
$$

which results in

$$
\sigma_{\mathrm{s}}^{2}=s^{\prime}(\mathbf{p}) /(N+P-M) .
$$

By substituting Equation 27, ABIC is reduced to

$$
\begin{aligned}
\mathrm{ABIC}= & (-2) \ln L\left(\sigma_{\mathrm{s}}^{2}, \sigma_{\mathrm{o}}^{2}, \sigma_{\mathrm{p}}^{2}\right) \\
= & (N+P-M)\left(\ln 2 \pi+\ln s^{\prime}\left(\mathbf{p}^{*}\right)-\ln (N+P-M)+1\right) \\
& +\ln \left\|\mathbf{E}^{\prime}\right\|-\ln \left\|\frac{1}{\beta^{2}} \mathbf{\Lambda}\right\|+\ln \left\|\mathbf{G}^{\mathrm{T}} \mathbf{E}^{\prime-1} \mathbf{G}+\frac{1}{\beta^{2}} \mathbf{L}^{\mathrm{T}} \mathbf{L}\right\| .
\end{aligned}
$$

As discussed in the main text, I adopt the hyperparameters that minimize ABIC in the inversion, performing minimization through a grid search. 